PROCEEDINGS OF THE

AMERICAN MATHEMATICAL SOCIETY

Volume 125, Number 2, February 1997, Pages 569-572

S 0002-9939(97)03480-1

\title{
REMARKS ON SPHERE-TYPE THEOREMS
}

\author{
HYEONG IN CHOI, SANG MOON KIM, AND SUNG HO PARK
}

(Communicated by Peter Li)

\begin{abstract}
We prove if $M$ is a complete Riemannian manifold with an embedded totally geodesic compact hypersurface $N$ such that $M$ has nonnegative sectional curvature, and the sectional curvature of $M$ is strictly positive in a neighborhood of $N$, then the pair $(M, N)$ is diffeomorphic to the pair $\left(S^{n}, S^{n-1}\right) / \pi_{1}(M)$. This result gives an affirmative answer to a question of $\mathrm{H}$. Wu in the case when $M$ is compact and simply connected.
\end{abstract}

\section{INTRODUCTION}

In [6], H. Wu obtained the following interesting sphere theorem.

Theorem A. Let $M$ be an n-dimensional simply connected compact Riemannian manifold with nonnegative sectional curvature. Let $N$ be an orientable totally geodesic compact hypersurface of $M$ such that the sectional curvature of $M$ is strictly positive in a neighborhood of $N$. Then the pair $(M, N)$ is homeomorphic to the standard pair $\left(S^{n}, S^{n-1}\right)$, where $S^{n-1}$ is regarded as the equatorial hypersurface in $S^{n}$.

His method of proof is the judicious use of Morse theory combined with the smoothing theory of convex functions developed by R. E. Greene and himself [4]. It is interesting to compare this kind of sphere theorem with the well-known sphere theorems which have the usual pinching condition of sectional curvature or some diameter information [1]. In his paper [6], Wu raised the question of whether $(M, N)$ might be in fact diffeomorphic to $\left(S^{n}, S^{n-1}\right)$. We found an affirmative answer to his question, and it is indeed the main objective of our paper to present the proof of this differentiable version of Wu's theorem.

We also obtained the non-simply-connected version by reducing the situation to the simply connected case with the use of the second variation argument of Frankel $[2]$.

\section{NotATIONS AND PRELIMINARIES}

We now present some relevant background materials. Let $M$ be a Riemannian manifold and $f: M \rightarrow \mathbb{R}$ be a continuous function. Let $\gamma:(-a, a) \rightarrow M$ be a

Received by the editors May 22, 1995.

1991 Mathematics Subject Classification. Primary 53C20.

Key words and phrases. Sphere theorem, Morse theory, convex function.

Supported in part by BSRI-94-1416, and by GARC.

(C)1997 American Mathematical Society 
geodesic such that $\gamma(0)=x \in M$ and $\dot{\gamma}(0)=X \in T_{x} M$. Define the following extended real numbers:

$$
\begin{gathered}
C f(x ; X)=\lim _{r \rightarrow 0} \inf \frac{1}{r^{2}}\{f(\gamma(r))+f(\gamma(-r))-2 f(\gamma(0))\}, \\
C f(x)=\inf C f(x ; X),
\end{gathered}
$$

where the infimum in (2) is taken over all unit vectors $X \in T_{x} M$.

Recall that $f$ is said to be strictly convex on an open set $U$ if there exists a strictly positive continuous function $\kappa$ on $U$ such that $C f \geq \kappa$ on $U$. If $f$ is $C^{2}$, then it is easy to see that $C f(x ; X)=D^{2} f(X, X)$, where $D^{2} f$ is the Hessian of $f$. The following smoothing theorem due to Greene and $\mathrm{Wu}$ [4] is our important tool.

Theorem B. Suppose $f$ is a strictly convex function on an open set $U$ in the above sense. Let $\xi$ be a strictly positive continuous function on $U$. Then there exists a strictly convex $C^{\infty}$ function $F$ on $U$ such that $|F-f|<\xi$.

Now let $M$ be as in Theorem A. Then $N$ disconnects $M$ into two components $M_{1}$ and $M_{2}$. Let us take the following convention: First, let $s(x)$ denote the signed distance from $x$ to $N$. The sign convention is chosen in such way that the points in $M_{1}$ (resp. $M_{2}$ ) are of positive (resp. negative) distance from $N$. Thus

$$
s(x)=\left\{\begin{array}{rll}
d(x, N) & \text { if } & x \in M_{1} \\
-d(x, N) & \text { if } & x \in M_{2} .
\end{array}\right.
$$

Using this signed distance, we define $U[a, b]=\{x \in M \mid a \leq s(x) \leq b\}$ for $a, b \in \mathbb{R}$. We also define $U[a, \infty)=\{x \in M \mid a \leq s(x)\}$. One can similarly define various sets like $U(a, b), U(a, \infty), U(a, b], U[a, b)$, etc., by adopting the notation convention for (half-) open or (half-) closed intervals. For $a \in \mathbb{R}$, we also define $N_{a}=\{x \in$ $M \mid s(x)=a\}$. Thus in this convention, $N=N_{0}$. For $\delta>0$, we also define $\rho_{+}^{\delta}$ : $U[-\delta, \infty) \rightarrow \mathbb{R}$ by $\rho_{+}^{\delta}(x)=d\left(x, N_{-\delta}\right)$, and we similarly define $\rho_{-}^{\delta}: U(-\infty, \delta] \rightarrow \mathbb{R}$ by $\rho_{-}^{\delta}(x)=d\left(x, N_{\delta}\right)$.

\section{Main Results}

Now we state our main result.

Theorem 1. Let $M$ be an n-dimensional simply connected compact Riemannian manifold with nonnegative sectional curvature. Suppose there exists an orientable totally geodesic compact hypersurface $N$ such that the sectional curvature of $M$ is strictly positive in a neighborhood of $N$. Then the pair $(M, N)$ is diffeomorphic to the standard pair $\left(S^{n}, S^{n-1}\right)$, where $S^{n-1}$ is regarded as the equatorial hypersphere in $S^{n}$.

Proof. Let us choose sufficiently small $\delta>0$ which satisfies the following:

(1) The sectional curvature of $M$ is strictly positive on $U[-2 \delta, 2 \delta]$.

(2) $N$ is diffeomorphic to all $N_{\alpha}$ for all $\alpha \in[-2 \delta, 2 \delta]$.

(3) $\rho_{+}^{\delta}$ is $C^{\infty}$ and $\nabla \rho_{+}^{\delta} \neq 0$ on $U(-\delta, 2 \delta)$.

(4) $\rho_{-}^{\delta}$ is $C^{\infty}$ and $\nabla \rho_{-}^{\delta} \neq 0$ on $U(-2 \delta, \delta)$.

(5) $\rho_{+}^{\delta}+\rho_{-}^{\delta}=2 \delta$ on $U[-\delta, \delta]$.

Let $k$ be a positive lower bound of the sectional curvature of $M$ on $U[-2 \delta, 2 \delta]$. Then there exists $\epsilon$ such that $0<\epsilon<\delta$ and $|S(X, X)| \leq \frac{\delta k}{2}|X|^{2}$ for all $X \in T N_{ \pm \epsilon}$, where $S$ is the second fundamental form of $N_{ \pm \epsilon}$. From now on, $\delta$ and $\epsilon$ are fixed. 
Claim. $-\log \rho_{+}^{\epsilon}\left(\right.$ resp. $\left.-\log \rho_{-}^{\epsilon}\right)$ is strictly convex on $U(\delta, \infty)($ resp. $U(-\infty,-\delta))$.

This claim follows from Wu's argument in [6, pp 533-534]. Repeating his argument and noticing the condition on $S$, we get

$$
C\left(-\log \rho_{+}^{\epsilon}(x ; X)\right) \geq \frac{k \delta}{2 b} \alpha^{2}+\frac{1}{b^{2}} \beta^{2},
$$

where $\alpha^{2}+\beta^{2}=1, X$ is a unit vector in $T_{x} M$ and $b=d\left(x, N_{-\epsilon}\right)$. We can then conclude that

$$
C\left(-\log \rho_{+}^{\epsilon}\right) \geq \min \left\{\frac{1}{b^{2}}, \frac{k \delta}{2 b}\right\}>0 .
$$

Thus the claim is proved.

Now define a function $\mu: U(\delta, \infty) \rightarrow \mathbb{R}$ by $\mu(x)=d\left(x, N_{\delta}\right)$. By the above claim and Theorem $\mathrm{B}$, there exists a strictly convex $C^{\infty}$ function $f_{1}$ on $U(\delta, \infty)$ such that $\left|f_{1}-\left(-\log \rho_{+}^{\epsilon}\right)\right|<\exp ^{-\frac{1}{\mu}}$ on $U(\delta, \infty)$.

Now define a function $\psi_{1}$ on $U\left[-\frac{\epsilon}{2}, \infty\right)$ by

$$
\psi_{1}(x)=\left\{\begin{array}{ccc}
f_{1}(x) & \text { if } & x \in U(\delta, \infty) \\
-\log \rho_{+}^{\epsilon}(x) & \text { if } \quad x \in U\left[-\frac{\epsilon}{2}, \delta\right] .
\end{array}\right.
$$

It is very important to note that $\psi_{1}$ is in fact $C^{\infty}$ on $U\left[-\frac{\epsilon}{2}, \infty\right.$ ). (The proof is straightforward by using the Newton divided difference formula.) We now use the standard Morse theory argument (see [6, pp 535-536], for example). Let $s_{\epsilon}=$ $\left.s\right|_{U\left[-\frac{\epsilon}{2}, \frac{\epsilon}{2}\right]}$ where $s$ is the the signed distance function defined in $\S 2$. Since $\delta>\epsilon$ are chosen sufficiently small, $U[-\epsilon, \epsilon]$ is diffeomorphic to $N \times[-\epsilon, \epsilon]$. For $p \in U[-\epsilon, \epsilon]$, let $\bar{p} \in N$ be the foot of $p$ on $N$. It is easy to see that $N$ is diffeomorphic to $S^{n-1}$ by [6], and we fix a diffeomorphism $\phi: N \rightarrow S^{n-1}$. Let $\pi(p)=\phi(\bar{p})$. Then the map $p \longmapsto\left(\pi(p), s_{\epsilon}(p)\right)$ gives a diffeomorphism $U[-\epsilon, \epsilon] \rightarrow S^{n-1} \times[-\epsilon, \epsilon]$. Now look at the vector field $Y_{1}=-k_{1} \frac{\nabla \psi_{1}}{\left|\nabla \psi_{1}\right|^{2}}$ on $U\left[-\frac{\epsilon}{2}, \infty\right)$, where $k_{1}$ is a constant to be chosen later. For $p \in U\left[-\frac{\epsilon}{2}, \infty\right)$, the flow line $\phi_{t}(p)$ through $p$ meets with $N$ at a unique point $\bar{p}$, and it is clear that $\bar{p}$ is the foot of $p$ on $N$. And the flow preserves the level sets $N_{\alpha}$, for $-\frac{\epsilon}{2} \leq \alpha \leq \frac{\epsilon}{2}$. Thus this fact, combined with Wu's argument, gives a diffeomorphism $\Psi_{1}$ of $U\left[-\frac{\epsilon}{2}, \infty\right)$ to a closed ball $B_{\eta_{1}} \subset \mathbb{R}^{n}$. We adjust the constant $k_{1}$ so that $N$ is mapped to the unit sphere $S^{n-1} \subset B_{\eta_{1}}$ and $\eta_{1}>1$.

As this flow preserves the level set $N_{\alpha}$, we can find constants $0<l_{1}<1<\eta_{1}$ and a strictly decreasing $C^{\infty}$ function $u_{1}:\left[-\frac{\epsilon}{2}, \frac{\epsilon}{2}\right] \rightarrow\left[l_{1}, \eta_{1}\right]$ with $u_{1}\left(-\frac{\epsilon}{2}\right)=\eta_{1}, u_{1}(0)=$ $1, u_{1}\left(\frac{\epsilon}{2}\right)=l_{1}$ so that, for $-\frac{\epsilon}{2} \leq \alpha \leq \frac{\epsilon}{2}, N_{\alpha}$ is diffeomorphically mapped to the concentric sphere $S^{n-1}\left(u_{1}(\alpha)\right)$ of radius $u_{1}(\alpha)$. Note also that the flow lines in $U\left[-\frac{\epsilon}{2}, \infty\right)$ correspond to the radial lines in $B_{\eta_{1}}$. Now, applying the same argument, we can find a $C^{\infty}$ function $\psi_{2}: U\left(-\infty, \frac{\epsilon}{2}\right] \rightarrow \mathbb{R}$ given by

$$
\psi_{2}(x)=\left\{\begin{array}{ccl}
f_{2}(x) & \text { if } & x \in U(-\infty,-\delta), \\
-\log \rho_{-}^{\epsilon}(x) & \text { if } \quad x \in U\left[-\delta, \frac{\epsilon}{2}\right],
\end{array}\right.
$$

where $f_{2}$ is a strictly convex $C^{\infty}$ function on $U(-\infty,-\delta)$. Let $Y_{2}=-k_{2} \frac{\nabla \psi_{2}}{\left|\nabla \psi_{2}\right|^{2}}$ on $U\left(-\infty, \frac{\epsilon}{2}\right]$, where $k_{2}$ is again a constant to be chosen later. Note that, even though the speeds are different, the flow lines of $Y_{1}$ and $Y_{2}$ coincide in $U\left[-\frac{\epsilon}{2}, \frac{\epsilon}{2}\right]$. Thus for $p \in U\left[-\frac{\epsilon}{2}, \frac{\epsilon}{2}\right]$, both flow lines meet $N$ at its foot $\bar{p} \in N$. Using the same argument as above, we can find a diffeomorphism $\Psi_{2}: U\left(-\infty, \frac{\epsilon}{2}\right] \rightarrow \tilde{B}_{\eta_{2}}$, another closed ball of radius $\eta_{2}>1$, such that the angular coordinates of $\Psi_{1}$ and $\Psi_{2}$ coincide on $U\left[-\frac{\epsilon}{2}, \frac{\epsilon}{2}\right]$. The radial part of $\Psi_{2}$ gives a strictly increasing $C^{\infty}$ function $u_{2}:\left[-\frac{\epsilon}{2}, \frac{\epsilon}{2}\right] \rightarrow\left[l_{2}, \eta_{2}\right]$ such that $u_{2}\left(-\frac{\epsilon}{2}\right)=l_{2}, u_{2}\left(\frac{\epsilon}{2}\right)=\eta_{2}$, and, adjusting the constant $k_{2}$, we can set 
$u_{2}(0)=1$, where $0<l_{2}<1<\eta_{2}$. Now there exists a strictly decreasing $C^{\infty}$ function $h:\left[l_{2}, \eta_{2}\right] \rightarrow\left[l_{1}, \eta_{1}\right]$ with $h\left(l_{2}\right)=\eta_{1}, h(1)=1$ and $h\left(\eta_{2}\right)=l_{1}$ such that $h \circ u_{2}(\alpha)=u_{1}(\alpha)$ for all $\alpha \in\left[-\frac{\epsilon}{2}, \frac{\epsilon}{2}\right]$.

Glue $B_{\eta_{1}}$ and $\tilde{B}_{\eta_{2}}$ by identifying the point $\left(r_{2}, \theta_{2}\right) \in \tilde{B}_{\eta_{2}}$ with $\left(r_{1}, \theta_{1}\right) \in B_{\eta_{1}}$, where $\theta_{1}=\theta_{2}$ and $r_{1}=h\left(r_{2}\right)$. This gluing process produces a manifold $\Sigma$ which is diffeomorphic to $S^{n}$, and our construction shows that our manifold $M$ is diffeomorphic to $\Sigma$. Moreover, the diffeomorphism of $\Sigma$ to $S^{n}$ can be chosen to map the image of $N$ to the equatorial hypersphere in $S^{n}$.

The following is a generalization of Theorem 1 in the case when $M$ is not simply connected.

Theorem 2. Let $M$ be a complete Riemannian manifold with an embedded totally geodesic compact hypersurface $N$. Suppose $M$ has nonnegative sectional curvature and the sectional curvature of $M$ is strictly positive in a neighborhood of $N$. Then the pair $(M, N)$ is diffeomorphic to the pair $\left(S^{n}, S^{n-1}\right) / \pi_{1}(M)$.

Proof. Let $\pi: \tilde{M} \rightarrow M$ be the universal covering space of $M$. Since $\pi^{-1}(N)$ has strictly positive sectional curvature, Bonnet's theorem implies that each component of $\pi^{-1}(N)$ has finite diameter, thus is compact. Then applying the second variation argument of Frankel [2], we see that $\pi^{-1}(N)$ cannot have more than one component. Thus $\pi^{-1}(N)$ is a compact connected finite covering manifold of $N$. Thus $\pi: \tilde{M} \rightarrow$ $M$ is a finite covering map, which implies that $\tilde{M}$ is compact. Then we can use Theorem 1 to complete the proof.

The following corollary is an easy consequence of our Theorem 1 and Theorem 2.

Corollary 1. Assume the conditions in Theorem 2 are valid.

(i) If $\operatorname{dim}(M) \geq 3$, then $i_{*}: \pi_{1}(N) \rightarrow \pi_{1}(M)$ is an isomorphism, where $i: N \rightarrow$ $M$ is the embedding.

(ii) If $\operatorname{dim}(M)=2$, then $i_{*}$ is onto.

\section{REFERENCES}

[1] J. Cheeger \& D. Ebin Comparison theorems in Riemannian Geometry, North-Holland, Amsterdam 1975. MR 56:16538

[2] T. Frankel On the fundamental group of a compact minimal submanifold, Annals of Math. $\mathbf{8 3}$ (1966),68-73 MR 32:4637

[3] R.E. Greene \& H. Wu $C^{\infty}$ approximation of convex, subharmonic, and plurisubharmonic functions, Ann. Sci. École Norm. Sup. (4) 12 (1979), 47-84. MR 80m:53055

[4] R.E. Greene \& H. Wu $C^{\infty}$ convex functions and manifolds of positive curvature, Acta Math. 137 (1976), 209-245. MR 56:16539

[5] H. Wu On certain Kähler manifolds which are q-complete, Proc. Symp. Pure Math. Amer. Math. Soc. 41 (1984), 253-276. MR 85j:32031

[6] H. Wu Manifolds of Partially Positive Curvature, Indiana Univ. Math. J. 36 (1987), 525548. MR 88k:53068

Department of Mathematics, Seoul National University, Seoul, 151-742 Korea

E-mail address: hichoi@math.snu.ac.kr 